\title{
Private Cloud Computing Platform Design Research Based on Network Virtualization Technology
}

\author{
Shihong Zhang ${ }^{1, \text { a }}$ \\ ${ }^{1}$ Department of Information Technology, Hainan Vocational College of Political Science and Law, \\ Haikou, 571100, China \\ aemail: qinhao3806@163.com
}

\begin{abstract}
Keywords: Private Cloud Services; Cloud Platform Flamework; Virtual Machine Deployment; Dynamic Allocation
\end{abstract}

\begin{abstract}
In order to increase the hardware resource utilization of the distributed cluster system, this paper, in combination with network virtualization technology, will put forward a private cloud platform implementation scheme based on a variety of flamework techniques. The scheme can focus the underlying hardware resources on realizing the on-demand segmentation, dynamic allocation and dynamic migration of the resources. It also proposes the virtual machine deployment mechanism which is based on dynamic allocation decision. In addition, the design has realized the private cloud computing service platform with good flexible and extensible performance, which can prove the feasibility and effectiveness of private cloud platform. The experimental results show that the dynamic allocation decisions can be deployed in a large amount of virtual machine, at the same time, it can keep the load balancing of private cloud platform.
\end{abstract}

\section{Introduction}

In recent years, along with the rapid development of computer technology, more and more computing services require the cloud computing [1]. Cloud computing has brought a variety of services for all walks of life, which greatly improves the equipment utilization, prevents the economic losses of the idle equipment and reduce the personnel and management cost [2], having a great temptation for enterprises. Therefore, building a flexible and efficient private cloud computing platform has become one of the highlights in the information technology enterprises at home and abroad. Nowadays, a private cloud platform technology has been more and more mature, in which business solutions and open source solutions are used to build a private cloud platform. Many business companies have provided relatively mature and overall solutions for private cloud, in which the typical one is IBM Blue Cloud Computing Platform [3]. Furthermore, open source solutions can also be used to build a private cloud platform, which is to use open source systems to build the private cloud platform of enterprises or organizations, typically including such open resources systems [4] as OpenStack and Eucalyptus.

Nowadays, Some development industries have strong application requirements for high performance computing. Although the small sign clusters like 32, 64 nodes have a good performance, they can not meet the increasing demand of large-scale computing tasks, which makes available resources in the idle state and has an urgent requirements to integrate the existing IT equipment foundation resources to the maximum, forming a unified, efficient and flexible private cloud computing platform and providing users a convenient and fast voluntary application and usage patterns. There are such problems in cloud construction pattern of commercial solutions as the high price, poor extensibility and complex authorization model. This is not suitable for usage in many cases. However, although the open source solution model has good scalability, it has a poor system compatibility, which gives less support for general service support, being unable to customize the cloud service module according to the actual needs.

Virtualization technology [5] is one of the core technology in cloud computing platform. Building private cloud computing platform based on virtualization technology can reduce the operational cost, simplify management and improve the system availability. The advantage of 
virtual technology depends on virtual resources deployment and the deployment effect depends on the service quality provided by the cloud platform to a large extent. Present studies on virtual machine deployment are less and mostly are based on the traditional deployment methods, which do not give full consideration to the load host machine can bear, leading to a bad match condition between host machine performance and load capacity produced by the virtual machines. It inevitably brings in the load imbalance problems due to unreasonable resources usage, which will affect the quality of service provided by cloud computing. According to the above two problems, this article is based on Java technology and it design and implement a flexible and extensible private cloud computing service platform, such as Infrastructure as a Service (IaaS), common resource front. The platform, which is based on computer cluster, a combination of the server. Through the server virtualization technology, it implements the on-demand segmentation, dynamic allocation and dynamic migration. It also proposes a dynamic allocation management mechanism, which can, to the extend, achieve the rapid deployment of the virtual machine resources, solve the load imbalance problem of cloud platform and improve the utilization of resources.

\section{Cloud Platform Framework Construction}

Platform overall architecture. The cluster structure in cloud computing environment is generally divided into two kinds: centralized and equations [6]. The private cloud plateform established in this paper regards the virtualization service as its core function, in which the most important physical resource support is the computer nodes and storage devices. In order to prevent the system crash caused by the single node, we use the equate resource structure. This architecture requires that each node of the whole network must have a complete service. When the new message arrives, the response node will have to spread the message to the various nodes to maintain the consistency of the entire network data. By analyzing platform function requirements and designing the system architecture of private cloud platform, this architecture can be divided into application layer, service layer and resource layer. Platform service layer is used to establish a set of generic service interface to communicate with local resources to. Resource layer can private shared resources for private cloud platform, including such resources as processor, memory and storage system. Network communication layer can provide the information exchange control for various data submitted by resource layer, including resource registration, verification and monitoring. These services will be regarded as an aid module of the virtual machine service to provide users with resource application. Application layer, through the development tool application programming interface (API), can invoke the corresponding service and then it can also use the corresponding resources to accomplish specific tasks.

Service formation design. On the basis of the above platform architecture, private cloud platform are divided into 7 service components according to the functional requirements. In the equate organization structure, each node has complete function, so each node has 7 function service components: user access interface (User Port), information service (Information), registration service(Register), task submission (Submit), file service (File), virtual machine deployment (Deployment) and task execution (Executor). The functional description of each service component is as follows:

User access interface. To provide users with access to the private cloud platform, the interface provides some functions such as monitoring platform running state, accessing to information resources and file system as well as applying for the virtual machine performing tasks.

Information services. It can maintain and monitor the normal operation of private cloud platform as well as gain the host running situation of each node.

Registration service. Cloud platform construction process, the dynamic state of applying for the virtual machine join to the network organization structure of private cloud platform.

Task submission. Receive the task request from the users, analyze and create the task description object and submit the task to the virtual machine node applied by users.

File service. Provide storage and transport the related documents, such as users' application for the image files in the virtual machine and the related document transmission to the execution node 
in the process of mission.

Virtual machine deployment. Analyze the specific requirements of users in the application for the virtual machine, evaluate the resource information in the information service, select the appropriate host and then deploy and start the virtual machine.

Task execution. Perform the task request submitted by the users in the virtual machine, monitor task status and feedback the results to the users.

The above seven functional components are not independent but close. On the basis of mutual collaboration, kinds of services functions of private cloud platform can be achieved. Private cloud platform system is consist of four modules such as information monitoring, file service, virtual machine service and operation service. Other service functions are based on the four modules and each module is mutually cooperated to meet the user's requirements for various functions.

Service component design. Spring enterprise framework technology simplify the organization, maintenance and management of the internal resources, which can perfectly combine with a variety of communication frameworks, shield the complex communication logic underlying and provide a wide range of optional service access methods to the external users to improve the system flexibility. Spring framework technology plays a key role in the process of service construction. Fig. 1 depicts the variety of combination methods of framework in the private cloud platform. Private cloud platform designed in this paper is based on the three frameworks (JRE, JETTY, Spring Container), constituting the basic service environment, on which every service component will be improved. When JETTY starts, Spring is responsible for the initialization of each application object and the realization of the dependency relationship between different objects. When the service is normal, Spring is responsible for receiving a request from the JETTY, parse and convert it into the corresponding method adjustment processing of the Resource layer and then return the results.

Regard Spring interface programming as the core method and combined with a variety of framework technology, it implements a development process depending on each service component. In the process of realization of some services, based on the idea of programming to an interface, we should firstly find out the methods provided by the service interface, and then clearify the entity class in Model layer according to the interface methods. The entity can be designed by the developers themselves or generally generated by the mentioned XSD tool (XML Schema Definition). The generated entities are the assurance of the success of the service. After finishing the above works, using the simple factory method to determine the implementation of the interface and it is managed by the Manager layer. The advantage is: when we need to modify a particular interface or use the new methods, we donnot need not change the original Imp 1. We just need to choose the new ways to reuse Manager, which increases the flexibility and scalability of the code, building the robust foundation for the later codes. At the same time, we should use Factory to choose the appropriate method to submit the Resource layer to the Logic layer. When the outer request arrives, the Resource layer can directly get through the Logic layer to choose the appropriate Imp l to conduct the specific tasks. 


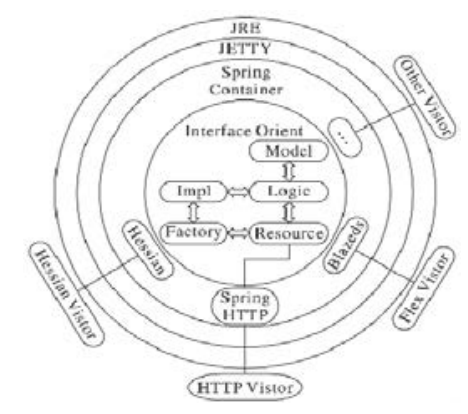

Fig.1. Private Cloud Platform Framework Combinations

\section{Virtual Machine Deployment Mechanism}

Virtual machine deployment process. Virtual machine resources deployment process in Cloud platform mainly include the following steps:

When API receives users' request, it will send the required virtual machine information to a private cloud platform control center, which can determine the correctness of the information. The virtual machine information includes the type of the virtual machine system, architecture, auditing, memory and other basic information.

Control center sends the virtual machine information to the image management center and looks for the image templates which meet the requirements. If finding the image template, we should copy the image files and return image location information to the control center; If not and no response, we should return the virtual machine template information which do not meet the requirements.

After control center receives image template location information, we should ask the network controller for allocation of fixed IP, MAC address and other related information, by which the corresponding IP and MAC address information will be generated and feedback to the control center.

Regarding the virtual machine dynamic allocation management decisions proposed in this paper as the distribution mode of the host node. After the distribution, control center will distribute the basic information of the virtual machine host node. At this moment, the virtual machine deployment is completed. Control center will send the user a feedback signal and the user can access the virtual machine after the start of the virtual machine.

When virtual machine starts, it will automatically load all service modules. The realization of the virtual machine rapid deployment requires the interaction of two modules between information monitoring module and file management module. Information monitoring module is responsible for collecting available nodes in the monitoring platform for the choice of host physical nodes. File transmission module is responsible for duplicating the template files in the image database to each physical node folder in Network File System (NFS). Supported by two functional modules, virtual machine management module can definite, start and deploy the virtual machine.

Virtual machine dynamic allocation. Resource description. In the private cloud platform, the structure of organization structure is based on the equation, so the management node can be any node of platform. To facilitate the management, we usually specify a node as the management node to take charge of managing the other nodes. This node will not be regarded as the deployment node of the virtual machine, it is only managing and monitoring the normal operation of the other nodes. The rest of the nodes can be regarded as virtual machine deployment node, which are called resource nodes(RS), whose node cluster is defined as:

$\mathrm{RS}=\{\mathrm{N} 1, \mathrm{~N} 2, \ldots . \mathrm{The} \mathrm{Nn}\}$

Private cloud platform uses NFS to share storage. Therefore, in general, the storage performance in the platform will be not considered and its storage management is mainly managed by NFS. The network between each node uses InfiniBand high-speed network, which is mainly aimed at the connection of the server, responsible for the communication between each node. It can improve the efficiency and the performance of the communication. NFS and InfiniBand can reduce the factors 
virtual machine deployment should consider, including the main properties of the node, such as the usage situation of CPU and the memory. Definition of the performance of the node is called th and its node's related services capabilities are defined as follows:

th $=\left\{\right.$ th $\left._{\text {proc }}, \mathrm{th}_{\text {mem }}, \mathrm{th}_{\mathrm{d}}\right\}$

Among them: th $\mathrm{proc}_{\text {, }}, \mathrm{th}_{\mathrm{mem}}$, th $\mathrm{h}_{\mathrm{d}}$ respectively present processor, memory and the load conditions of the hard disk.

Virtual machine dynamic allocation mechanism. Dynamic allocation management of virtual machine will determine whether the private cloud platform can run steadily. Reasonable allocation mechanism can real-timely monitor the running status of all deployment node and do timely adjustment according to the specific situation to keep a balance of system load. Virtual machine management module can monitor the request of a newly-built virtual machine from a user, analyze each performance index of the virtual machine, such as the number of CPU cores, memory size, etc., and select the optimal reservoir physical nodes through the dynamic allocation decision algorithm.

If the node can be found through the decision-making, the virtual machine image will be created and fixed into the node according to the above mentioned deployment steps and started to join a private cloud platform; If the suitable physical nodes have not been found, it shows that the resource of the private cloud platform physical nodes is of scarcity, which has reached full-load condition. After the start of private cloud platform, the monitoring module will follow it and real-timely monitor the usage situation of each physical node. The upper limit value of the formula threshold is defined as "th" and the lower limit value is defined as $t_{\min }$. When the threshold of monitoring virtual machine is below th $\mathrm{m}_{\min }$, it suggests that the virtual machine is in idle situation. When a node' threshold is detected to exceed “th”, private cloud platform will do some processing to the node. Specific implementation process is as follows:

If the virtual machine in this node is loaded, it suggests that virtual machine is in online migration. In this position, we will choose a node with larger load container or higher requirements for its performance. An optimal host will be chosen through the virtual machine allocation decision method.

If the host migrated can be found, then the selected virtual machine will be online migrated into the node. And in the process of migration, the running program in the virtual machine will not be affected. If not, we will continue to examine the unused virtual machine in the physical nodes.

After finding the spare virtual machine and if the released resource performance can support to migrate a virtual machine, the unused virtual machine will be destructed and started to do the online migration.

Virtual machine dynamic allocation decisions. Decision allocation of virtual machines is different from the task of platform, for the virtual machine deployment time is relatively fixed and controllable. The running time of a virtual machine in the platform nodes is generally longer. When the node hardware resource is sufficient, it is not generally happened to over-interfere the operation of the virtual machine. Therefore, It is crucial to choose a better physical node to deploy. Distribution and the decision-making process mainly include the deployment of a single virtual machine and multiple virtual machine.

\section{Conclusion}

On the basis of the further study of cloud computing architecture and virtualization technology characteristics, we can implement a private cloud platform which can provide the infrastructure as a service function to the outer with the combination of a variety of framework design. The platform integrates the distributed system hardware resources, improve the resource usage efficiency and proposes a virtual machine dynamic allocation strategy due to inevitable load imbalance prolem. According to the characteristics of the virtual machine resources and combined with the existing physical node load situation, this strategy has carried on the dynamic deployment to the virtual machine and completed the dynamic allocation of virtual machine to achieve a better balance in the whole load of the private cloud platform. With Fourier finite difference prestack depth migration as the application background, it uses the Marmousi model application as an example to prove the 
feasibility of private cloud platform and virtual machine operation and do the virtual machine deployment tests. The experimental results show that using the proposed dynamic allocation decisions can allocate a large amount of virtual machine on keep a better load balancing of private cloud platform.

\section{Reference}

[1] ARMBRUST M, FOX A, GRIFFITH R, et al. A view of cloud computing [J]. Communications of the ACM, 2010, 53 (4): 50 -58.

[2] YE K, WU Z. Power management of virtualized cloud computing platform [J]. Chinese Journal of Computers, 2012, 35(6): 1263 -1280.

[3] FANG W, WEN X, PAN W, et al. Cloud computing: conceptions, key technologies and application [J]. Journal of Nanjing University of Information Science and Technology: Natural Science Edition, 2012, 4(4): 351-361.

[4] JIANG Y, WANG W, CAO L, et al. Construction of private cloud computing platform based on open source software [J]. Telecom Science, 2013, 29(1): 68-75.

[5] XU W, MADISON K, FLINN M, et al. Applying virtualization technology in security education [J]. Procedia —-Social and Behavioral Sciences, 2014, 141: 10-14.

[6] COFFMAN E G. Computer and job-shop scheduling theory [M]. Hoboken: John Wiley and Sons, 1976: 233-251. 